This item was submitted to Loughborough's Research Repository by the author.

Items in Figshare are protected by copyright, with all rights reserved, unless otherwise indicated.

Higher mode sound transmission from a point source through a rectangular aperture

PLEASE CITE THE PUBLISHED VERSION

http://dx.doi.org/10.1121/1.3519189

PUBLISHER

(C) Acoustical Society of America

VERSION

AM (Accepted Manuscript)

LICENCE

CC BY-NC-ND 4.0

REPOSITORY RECORD

Horner, Jane L., and K.S. Peat. 2011. "Higher Mode Sound Transmission from a Point Source Through a Rectangular Aperture”. figshare. https://hdl.handle.net/2134/8296. 
This item was submitted to Loughborough's Institutional Repository (https://dspace.lboro.ac.uk/) by the author and is made available under the following Creative Commons Licence conditions.

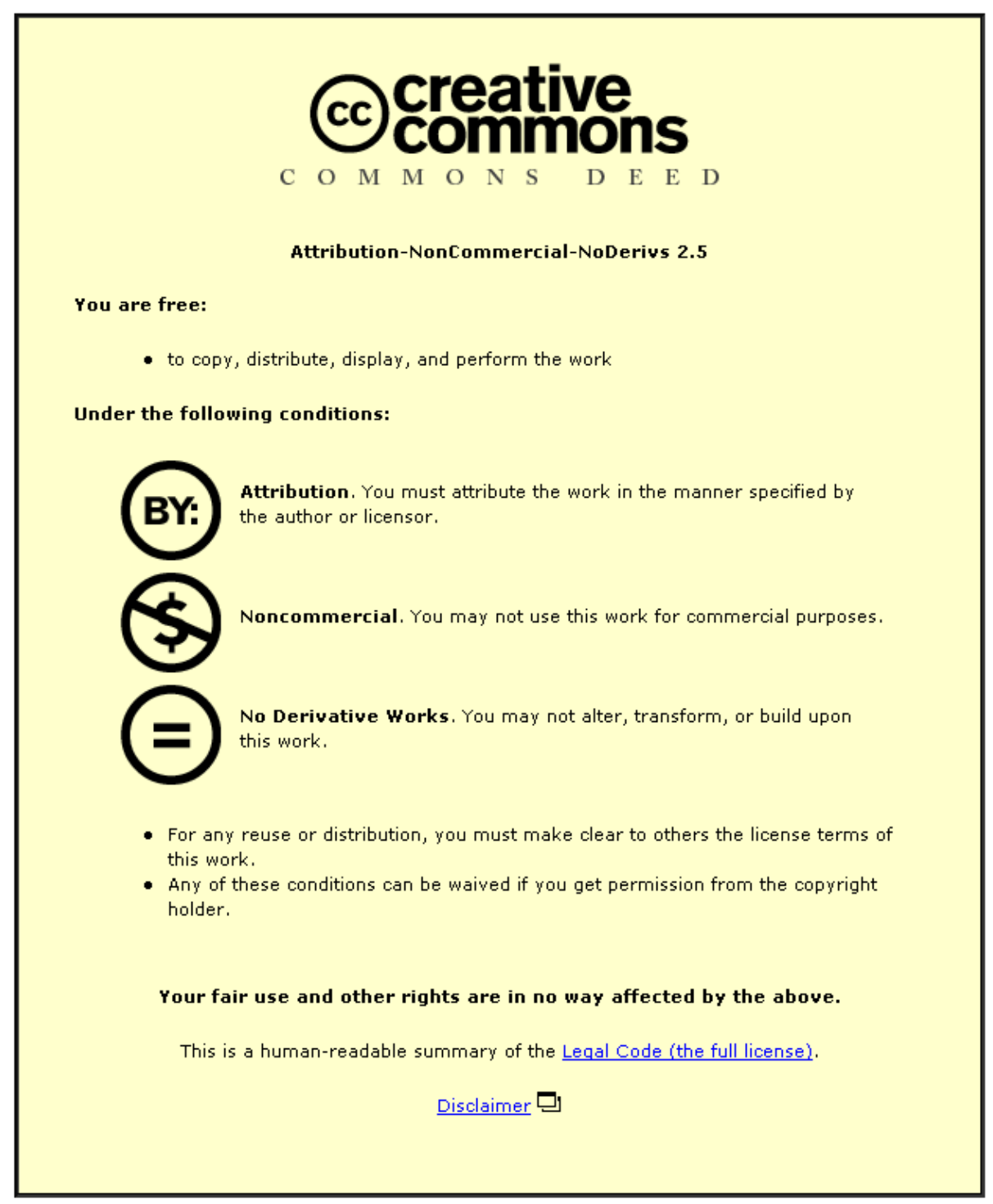

For the full text of this licence, please go to: http://creativecommons.org/licenses/by-nc-nd/2.5/ 


\title{
Higher mode sound transmission from a point source through a rectangular aperture
}

\author{
J. L. Horner ${ }^{a)}$ and K. S. Peat \\ Department of Aeronautical and Automotive Engineering, Loughborough University, Loughborough, \\ LE11 3TU, United Kingdom
}

(Received 29 June 2010; revised 31 October 2010; accepted 2 November 2010)

\begin{abstract}
This paper considers the higher-order scattered and transmitted wave fields that result when an acoustic wave from a point source impinges at an arbitrary angle on a rectangular aperture in a rigid, thick wall. In this analysis, it is assumed that free field conditions exist on both sides of the aperture. Although the full scattered and transmitted pressure fields contain both modal sum and modal coupling effects, the modal coupling effects of the higher-order modes are ignored such that an approximate analytical solution to the uncoupled analysis can be utilized. Experiments have been undertaken to measure the sound pressure levels in the transmitted field that result when sound from a point source impinges on the opposite side of a rectangular aperture. Measurements were made with the source located at the required position to drive a particular in-aperture higher-order mode. The source was also located at positions that did not directly excite any in-aperture higher-order mode at a cut-on frequency. These results indicate that the approximate analysis developed here gives accurate solutions whether or not any mode of the aperture is driven at cut-on. Thus, the method can be used for any relative location of a source from a rectangular aperture of any dimensions.
\end{abstract}

(C) 2011 Acoustical Society of America. [DOI: 10.1121/1.3519189]

PACS number(s): 43.20.Fn [AMJD]

Pages: $5-11$

\section{INTRODUCTION}

The transfer of acoustic energy from one domain to another via an opening or aperture is a common problem in many engineering applications. Quite commonly such apertures are rectangular in cross-section and have a thickness that is not negligible in comparison to a wavelength of the impinging sound. Thus, for a solution over a wide frequency range, it is necessary to consider the contribution from higher-order modes. For plane-wave impingement at low frequencies, or small Helmholtz numbers, when the wavelength of the incident wave is much greater than the dimensions of the rectangular aperture, there exist established approximate methods to estimate the sound fields, such as that developed by Sauter and Soroka. ${ }^{1}$ At higher frequencies, when the wavelength of the incident plane wave approaches the dimensions of the aperture, higher-order duct modes will propagate in the aperture. Thus, full solutions to the problem must include coupling between these higher-order modes, ${ }^{2}$ but this results in a fully numerical analysis that is computationally intensive. There has been previous work that evaluates the fully coupled problem of plane-wave impingement on a rectangular aperture over a wide frequency range, see for example Refs. 3-5.

In particular, Park and Eom ${ }^{5}$ used an approach utilizing the Fourier transform and mode matching to obtain a rigorous solution to the fully coupled problem, with improved numerical efficiency as compared with earlier methods. Horner and Peat ${ }^{6}$ developed this approach to determine a simple approximate solution for the scattered field from a rectangular aperture in a rigid baffle separating two semi-infinite half-spaces

\footnotetext{
a) Author to whom correspondence should be addressed. Electronic mail: J.L.Horner@lboro.ac.uk
}

when the frequency of excitation is such that higher-order modes freely propagate in the aperture. All analysis was undertaken in non-dimensional wave number space to allow results to be applicable to any combination of aperture size and frequency. It was shown that only a relatively small number of modes make a significant contribution to a given solution. Furthermore, it was found that when the incident plane wave excites an aperture mode at its cut-on frequency, the error in using an uncoupled solution is insignificant. An analytical approximation to the self-coupling coefficients was developed that resulted in approximate analytical expressions for all of the field potentials.

In this paper, the previous theoretical work ${ }^{6}$ is extended to consider wave impingement on a rectangular aperture from a point source in Sec. II. The development of a point source analysis enabled a more direct approach to the experimental investigation of the scattered field, as it did not require the construction of a plane-wave source. In Sec. III, details are given of the experimental work to measure the sound pressure that results on the transmitted side when sound from a point source is incident upon a rectangular aperture. This measured data is compared with theoretical results from the approximate uncoupled analytical solution in Sec. IV. Testing was undertaken with the point source located to drive a particular higher-order mode exactly at cut-on. Also the source was a located at positions that did not relate to the precise cut-on condition of any specific high-order mode. Due to practical restrictions, only the $(1,0)$, $(2,0)$, and $(2,1)$ were measured; however, this did allow for both axial and tangential modes to be investigated. Good agreement between measured and predicted results was found not only when the impinging wave excites one mode of the aperture exactly at cut-on, as might have been expected from the previous theoretical investigation ${ }^{6}$ but 
also for any randomly chosen impinging wave. This observation greatly extends the practical use of the approximate solutions given here and earlier. ${ }^{6}$ Although measured data has not been presented for a plane-wave source, it is reasonable to assume that the comparison would be similar to the point source results.

\section{DESCRIPTION OF ACOUSTIC FIELDS}

The analysis generally follows that given by Park and Eom $^{5}$ for an incident plane wave where full details can be found. A summary of the analysis is given here with details reserved for where the present analysis differs due to the assumed point source of sound. The scheme for non-dimensionalization follows that of Horner and Peat ${ }^{6}$ and serves to generalize and clarify the analysis.

Consider a rectangular aperture of size $2 a \times 2 b$ in a rigid wall of thickness $d$ which is of infinite extent (Fig. 1). Let the aspect ratio be $A=a / b$ and the depth ratio be $D=d / a$. Let

$$
\Phi=\Phi^{p}+\Phi^{r}+\Phi^{s} \quad \text { in } \quad z \geq 0,
$$

where the velocity potential for a spherical wave from the point source $P$ of wave number $k$ may be written as

$$
\Phi^{p}(x, y, z)=\frac{a}{\left|\underline{R}_{p}\right|} e^{i k\left|\underline{R}_{p}\right|},
$$

where $\underline{R}_{p}$ is the vector from point source $P$ to general position $(x, y, z)$. A time base of $e^{-i \omega t}$ is suppressed throughout for brevity. The resulting reflected and scattered velocity potentials are given by

$$
\begin{aligned}
\Phi^{r}(x, y, z)= & \frac{a}{\left|\underline{R}_{r}\right|} e^{i k\left|\underline{R}_{r}\right|}, \\
\Phi^{S}(x, y, z)= & \frac{1}{4 \pi^{2}} \int_{-\infty}^{\infty} \int_{-\infty}^{\infty} \tilde{\Phi}^{s}(\zeta a, \eta b) \\
& \times e^{-i(\zeta x+\eta y-\kappa z)} d(\zeta a) d(\eta b),
\end{aligned}
$$

where source $R$ is the image of source $P$ about $z=0$, and $\kappa$ is the scattered Helmholtz number. $\tilde{\Phi}^{s}(\zeta, \eta)$ is the Fourier transform of $\Phi^{s}(x, y, 0), \quad(\kappa a)=\sqrt{(k a)^{2}-(\zeta a)^{2}-(\eta b A)^{2}}$,

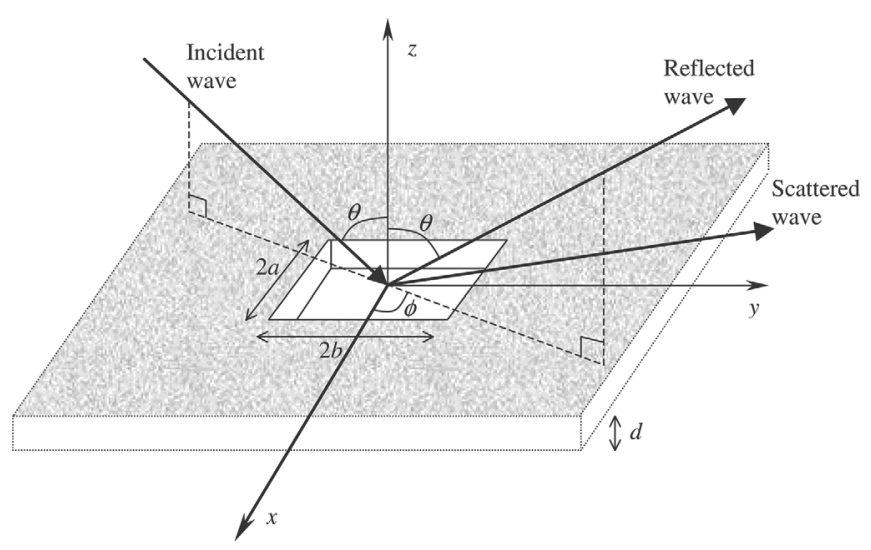

FIG. 1. Sketch of aperture. and $\zeta, \eta$ are the recoil wave numbers. Inside the duct the field is given over the depth, $d$, by

$$
\begin{aligned}
\Phi^{d}(x, y, z)= & \sum_{m=0}^{\infty} \sum_{n=0}^{\infty}\left[c_{m n} \cos \xi_{m n} d\left(\frac{z}{d}+1\right)\right. \\
& \left.+d_{m n} \sin \xi_{m n} d\left(\frac{z}{d}+1\right)\right] \cos \frac{m \pi}{2}\left(\frac{x}{a}+1\right) \\
& \times \cos \frac{n \pi}{2}\left(\frac{y}{b}+1\right)
\end{aligned}
$$

where $\left(\xi_{m n} d\right)=D\left(\xi_{m n} a\right)=D \sqrt{(k a)^{2}-(m \pi / 2)^{2}-(n \pi A / 2)^{2}}$, and $\xi_{m n} d$ is an in-aperture axial Helmholtz number.

It is assumed that a rigid wall of infinite extent surrounds the aperture on the surfaces of which the normal component of the velocity potential gradient must be zero. Since source $R$ is the image of source $P$ about $z=0$, it follows that on $z=0, \partial \Phi^{p} / \partial z+\partial \Phi^{r} / \partial z=0$ everywhere, $\partial \Phi^{s} / \partial z=\partial \Phi^{d} / \partial z$ over the aperture and $\partial \Phi^{s} / \partial z=0$, elsewhere. Thus 5,6

$$
\begin{aligned}
\tilde{\Phi}^{s}(\zeta a, \eta b)= & -i \frac{1}{(\kappa a)} \sum_{m=0}^{\infty} \sum_{n=0}^{\infty}\left(\xi_{m n} a\right)\left[c_{m n} \sin \left(\xi_{m n} d\right)\right. \\
& \left.-d_{m n} \cos \left(\xi_{m n} d\right)\right] G_{m}(\zeta a) G_{n}(\eta b),
\end{aligned}
$$

where

$$
G_{m}(u)=\frac{u\left\lfloor(-1)^{m} e^{i u}-e^{-i u}\right\rfloor}{u^{2}-(m \pi / 2)^{2}} .
$$

Consider next the continuity of potential boundary condition on the upper edge of the aperture

$$
\begin{aligned}
& \Phi^{p}(x, y, 0)+\Phi^{r}(x, y, 0)+\Phi^{s}(x, y, 0) \\
& =\Phi^{d}(x, y, 0) \text { on }|x| \leq a,|y| \leq b .
\end{aligned}
$$

Substitution from Eqs. (2)-(6), followed by multiplication by $\cos (p \pi / 2)(x / a+1) \cos (q \pi / 2)(y / b+1)$ and integration over the aperture yields $s^{5,6}$

$$
\begin{array}{r}
\gamma_{p q}+\frac{i}{(2 \pi)^{2}} \sum_{m=0}^{\infty} \sum_{n=0}^{\infty}\left[c_{m n} \sin \left(\xi_{m n} d\right)-d_{m n} \sin \left(\xi_{m n} d\right)\right] I_{m n p q} \\
=\varepsilon_{p} \varepsilon_{q}\left[c_{p q} \cos \left(\xi_{m n} d\right)+d_{p q} \sin \left(\xi_{p q} d\right)\right]
\end{array}
$$

where $\varepsilon_{0}=2, \varepsilon_{1}=\varepsilon_{2}=\cdots=1$,

$$
\begin{aligned}
I_{\text {mnpq }}= & \left(\xi_{m n} a\right) \int_{-\infty}^{\infty} \int_{-\infty}^{\infty} \frac{1}{(\kappa a)} G_{m}(\zeta a) G_{p}(-\zeta a) \\
& \times G_{n}(\eta b) G_{q}(-\eta b) d(\zeta a) d(\eta b)
\end{aligned}
$$

and

$$
\begin{aligned}
\gamma_{p q}= & 2 \int_{-1}^{1} \int_{-1}^{1} \Phi^{p}(x, y, 0) \cos \frac{p \pi}{2}\left(\frac{x}{a}+1\right) \\
& \times \cos \frac{q \pi}{2}\left(\frac{y}{b}+1\right) d\left(\frac{x}{a}\right) d\left(\frac{y}{b}\right) .
\end{aligned}
$$


In the transmitted field $(z<-d)$ let

$$
\begin{aligned}
\Phi & =\Phi^{t}(x, y, z) \\
& =\frac{1}{4 \pi^{2}} \int_{-\infty}^{\infty} \int_{-\infty}^{\infty} \tilde{\Phi}^{t}(\zeta a, \eta b) e^{-i[\zeta x+\eta y+\kappa(z+d)]} d(\zeta a) d(\eta b) .
\end{aligned}
$$

Application of the boundary conditions for continuity of potential and its normal derivative on the lower surface $z=-d$ and similar analysis to that above yield

$\tilde{\Phi}^{t}(\zeta a, \eta b)=-i \frac{1}{(\kappa a)} \sum_{m=0}^{\infty} \sum_{n=0}^{\infty}\left(\xi_{m n} a\right) d_{m n} G_{m}(\zeta a) G_{n}(\eta b)$

and

$$
\frac{i}{(2 \pi)^{2}} \sum_{m=0}^{\infty} \sum_{n=0}^{\infty} d_{m n} I_{m n p q}=\varepsilon_{p} \varepsilon_{p} c_{p q} .
$$

If cross-coupling of modes is ignored and the approximation ${ }^{6}$

$$
I_{m n m n} \approx 4 \pi^{2} \varepsilon_{m} \varepsilon_{n}
$$

is employed, then it follows from Eq. (12) that

$$
c_{m n} \approx i d_{m n},
$$

which implies the phase relationship between the two inaperture waves may be considered to be independent of the mode. It now follows from Eq. (9) that

$$
d_{m n}=\gamma_{m n} /\left\{2 \varepsilon_{m} \varepsilon_{n}\left[\sin \left(\xi_{m n} d\right)+i \cos \left(\xi_{m n} d\right)\right]\right\} .
$$

Substituting Eqs. (13) and (17) into Eq. (12) gives

$$
\begin{aligned}
\Phi^{t}(x, y, z)= & \frac{i}{8 \pi^{2}} \sum_{m=0}^{\infty} \sum_{n=0}^{\infty} \frac{\gamma_{m n}\left(\xi_{m n} a\right)}{\varepsilon_{m} \varepsilon_{n}\left[\sin \left(\xi_{m n} d\right)+i \cos \left(\xi_{m n} d\right)\right]} \\
& \times \int_{-\infty}^{\infty} \int_{-\infty}^{\infty} \frac{1}{(\kappa a)} G_{m}(\zeta a) G_{n}(\eta b) e^{-i[\zeta x+\eta y+\kappa(z+d)]} \\
& \times d(\zeta a) d(\eta b) .
\end{aligned}
$$

Now it follows from Eqs. (2) and (11) that

$$
\begin{aligned}
\gamma_{m n}= & 2 \int_{-1}^{1} \int_{-1}^{1} \frac{a}{|\underline{R} p|} e^{i k\left|\underline{R}_{p}\right|} \cos \frac{m \pi}{2}\left(\frac{x}{a}+1\right) \\
& \times \cos \frac{n \pi}{2}\left(\frac{y}{b}+1\right) d\left(\frac{x}{a}\right) d\left(\frac{y}{b}\right)
\end{aligned}
$$

where if the source is centered at $\left(-X_{o},-Y_{o}, Z_{o}\right)$, then

$$
\left|\underline{R}_{p}\right|^{2}=\left(X_{o}+x\right)^{2}+\left(Y_{o}+y\right)^{2}+Z_{o}^{2} .
$$

It is assumed that the source is relatively far from the aperture, such that $a, b \ll R_{o}$, where $R_{o}^{2}=X_{o}^{2}+Y_{o}^{2}+Z_{o}^{2}$. Then, over the aperture

$$
\left|\underline{R}_{p}\right| \approx R_{o}\left(1+\frac{x X_{o}}{R_{o}^{2}}+\frac{y Y_{o}}{R_{o}^{2}}\right)
$$

such that Eq. (19) becomes

$$
\begin{aligned}
\gamma_{m n}= & \frac{2 e^{i k R_{o}}}{\left(R_{o} / a\right)} \int_{-1}^{1} \int_{-1}^{1} e^{i\left(k a X_{o} / R_{o}\right)(x / a)} e^{i\left(k b Y_{o} / R_{o}\right)(y / b)} \\
& \times\left(1-\frac{X_{o}}{R_{o}^{2}} x-\frac{Y_{o}}{R_{o}^{2}} y\right) \cos \frac{m \pi}{2}\left(\frac{x}{a}+1\right) \\
& \times \cos \frac{n \pi}{2}\left(\frac{y}{b}+1\right) d\left(\frac{x}{a}\right) d\left(\frac{y}{b}\right)
\end{aligned}
$$

Let

$$
\frac{k a X_{o}}{R_{o}}=\frac{p \pi}{2}, \quad \frac{k b Y_{o}}{R_{o}}=\frac{q \pi}{2},
$$

where $p$ and $q$ are generally non-integer values, unlike $m$ and $n$. If $p$ and $q$ are both integers then the source location is such that the source is tuned to drive mode $p, q$ within the aperture at cut-on. Using Eq. (23), Eq. (22) simplifies to

$$
\begin{aligned}
\gamma_{m n}= & \frac{-2 e^{i k R_{o}}}{\left(R_{o} / a\right)}\left\{G_{m}\left(\frac{p \pi}{2}\right) G_{n}\left(\frac{q \pi}{2}\right)-\frac{i}{k R_{o}}\right. \\
& \left.\times\left[G_{m}\left(\frac{p \pi}{2}\right) H_{n}\left(\frac{q \pi}{2}\right)+G_{n}\left(\frac{q \pi}{2}\right) H_{m}\left(\frac{p \pi}{2}\right)\right]\right\},
\end{aligned}
$$

where

$$
H_{\mu}(u)=u \int_{-1}^{1} \theta e^{i u \vartheta} \cos \frac{\mu \pi}{2}(\theta+1) d \theta= \begin{cases}-i F_{\mu}(u)+\frac{\left[u^{2}+(\mu \pi / 2)^{2}\right]}{\left[u^{2}-(\mu \pi / 2)^{2}\right]} G_{\mu}(u), & u \neq \pm \mu \pi / 2, \\ -(i / 2) e^{\mp i(\mu \pi / 2),} & u= \pm \mu \pi / 2, \quad \mu \neq 0 \\ 0, & u=\mu \pi / 2=0\end{cases}
$$

and

$$
F_{\mu}(u)=\frac{u\left\lfloor(-1)^{\mu} e^{i u}+e^{-i u}\right\rfloor}{u^{2}-(\mu \pi / 2)^{2}} .
$$

Following substitution from Eq. (24) into Eq. (18), one can evaluate the acoustic potential at any location in the transmitted field, given the location of the source relative to the aperture and the geometry of the aperture.

Finally, since the source has already been assumed to be relatively far from the aperture, then at a position close to the source, the acoustic potential is essentially that of the point source only, as the contributions from the reflected and 
scattered fields will be negligible. Thus, from Eq. (1), $\Phi$ $\approx \Phi^{p}$. Let the acoustic pressure at a small distance $\left|\underline{R}_{p}\right|$ from the source be $p^{p}$ and the acoustic pressure at some general point in the transmitted field be $p^{t}$, then

$$
p^{t} \approx p^{p} \Phi^{t} / \Phi^{p} .
$$

Hence, if the acoustic pressure is measured at a small distance $\left|\underline{R}_{p}\right|$ from the source, then the acoustic pressure at any location in the transmitted field can be evaluated from Eqs. (2), (18), and (25).

\section{EXPERIMENTAL SETUP}

To achieve the desired conditions of a point source exciting an aperture separating two free fields, a loudspeaker within a fully anechoic chamber was used as the source. The main doors into the anechoic chamber from the outdoors were kept open, and a rigid baffle plate was constructed in the rectangular doorway of dimensions $2.97 \mathrm{~m} \times 2.39 \mathrm{~m}$ in the vertical ( $x$ direction) and horizontal ( $y$ direction) directions, respectively. This prevented any sound propagating around the sides of the baffle. The baffle plate was constructed of a rigid foam core with plastic covering on both sides, to give a total thickness of $28 \mathrm{~mm}$. A rectangular aperture of size $1.54 \mathrm{~m} \times 1.03 \mathrm{~m}$ in the $x$ and $y$ directions was left in the baffle plate. Sound level meters were used to measure the sound pressure level of both the incident wave upon the aperture at a distance of $1 \mathrm{~m}$ from the loudspeaker source inside the anechoic chamber and also at a variety of positions in the transmitted field on the outdoor side of the baffle plate. Measurements in the transmitted field were made over arcs of radius $5 \mathrm{~m}$ in a horizontal plane centered on a vertical line through the center of the orifice, hence at constant vertical heights above ground. The measurement planes used were at heights above ground of $1.48,1$, and $0.65 \mathrm{~m}$, corresponding to $x=-0.46,-0.94$, and $-1.29 \mathrm{~m}$, respectively. Measurements were taken at $5^{\circ}$ intervals over an arc of $\pm 25^{\circ}$. The maximum angle of the measurement arc was restricted by the fact that the doors of the anechoic chamber could only be opened so far as to be perpendicular to the baffle plate.

(a)

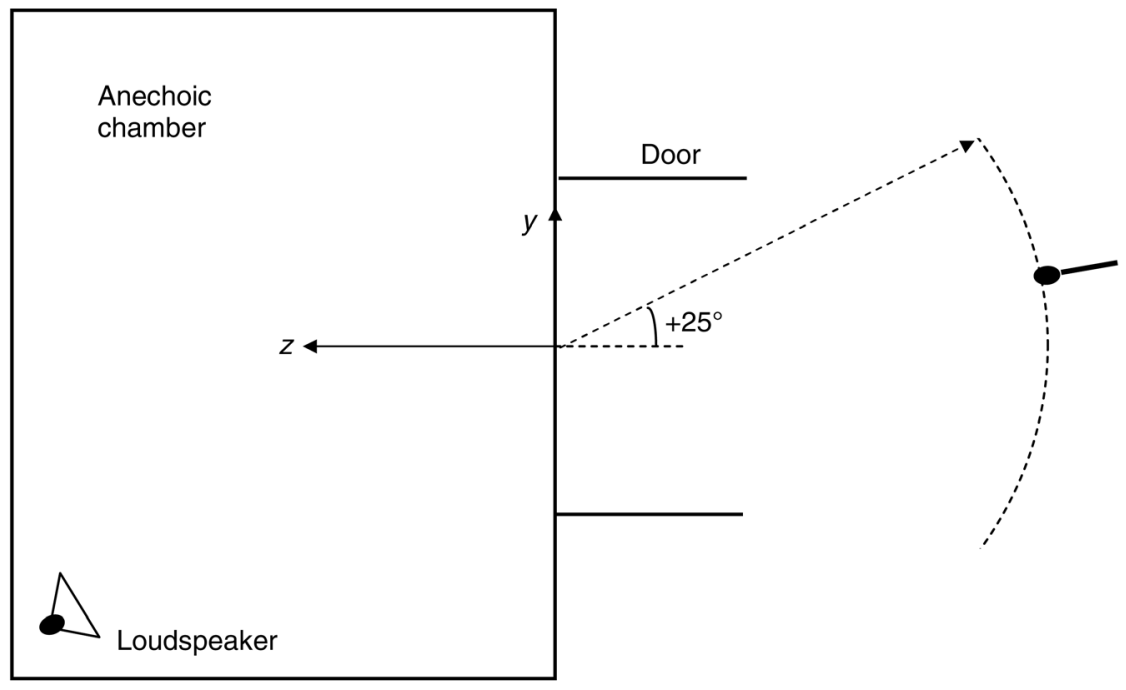

FIG. 2. Sketch of experimental setup. (a) Plan view and (b) side view.

(b)

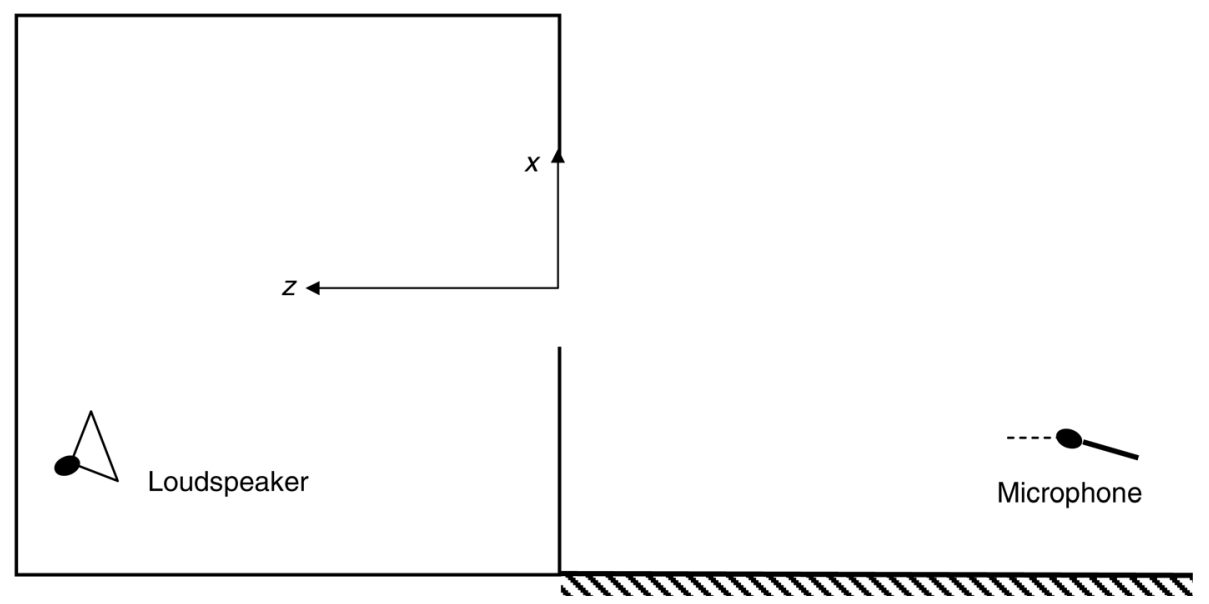




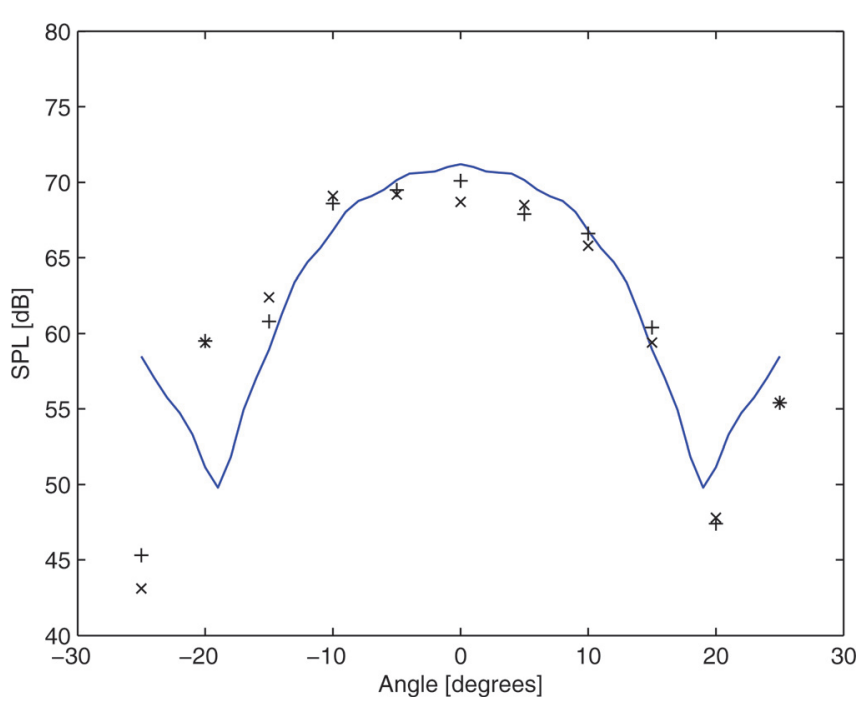

FIG. 3. (Color online) SPL in the transmitted field at $x=-0.46$. Loudspeaker positioned at $p=1, q=0 .+\times$ Experimental; — theoretical.

The doors were lined with thick foam to reduce errors from reflections. Thick foam was also placed on the ground outside the anechoic chamber to reduce reflection of sound from the ground plane to the sound level meter in the transmitted field. Figure 2 shows sketches of the setup in terms of both a side and a plan view in planes through the center of the orifice.

The loudspeaker was positioned within the plane $z=6.6 \mathrm{~m}$, as far distant from the orifice as was practicable and within the negative $x-y$ quadrant. A signal generator was used to input a $1 \mathrm{kHz}$ sine wave to the speaker for all tests, which gave of the order of 25 higher-order modes cut-on in the aperture. Sound pressure level measurements were made in the $1 / 3$ octave band centered on $1 \mathrm{kHz}$. The sound pressure level $1 \mathrm{~m}$ distant from the loudspeaker was close to $100 \mathrm{~dB}$ for all tests. Levels of background noise on the transmitted side of the orifice were consistently measured as $40 \mathrm{~dB}$ or less in normal conditions under which measurements were retained. Generally, the recorded levels of sound transmission through the aperture were $50 \mathrm{~dB}$ or greater such that the background noise would

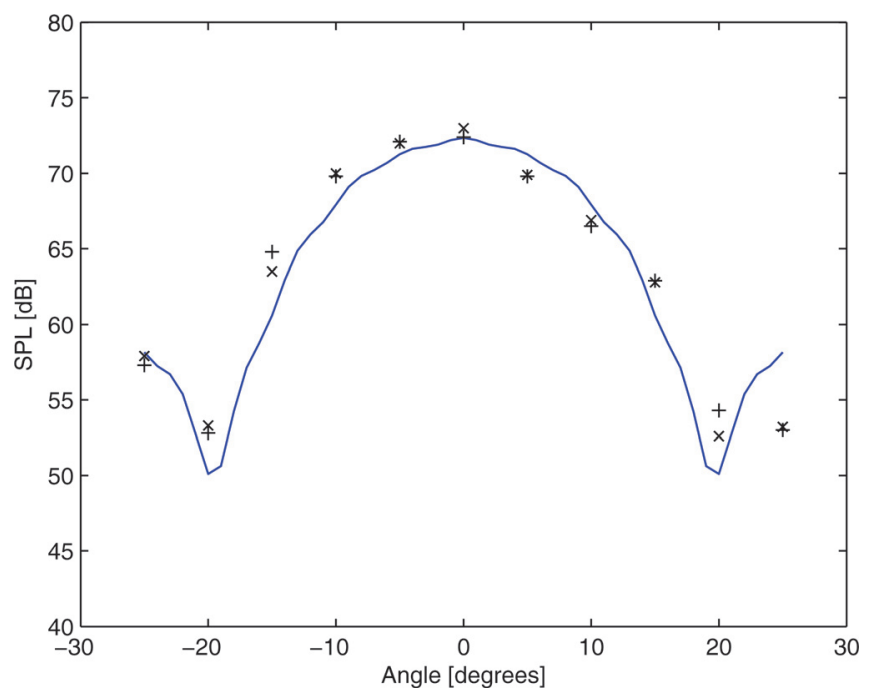

FIG. 4. (Color online) SPL in the transmitted field at $x=-0.46$. Loudspeaker positioned at $p=2, q=0 .+\times$ Experimental; $\longrightarrow$ theoretical.

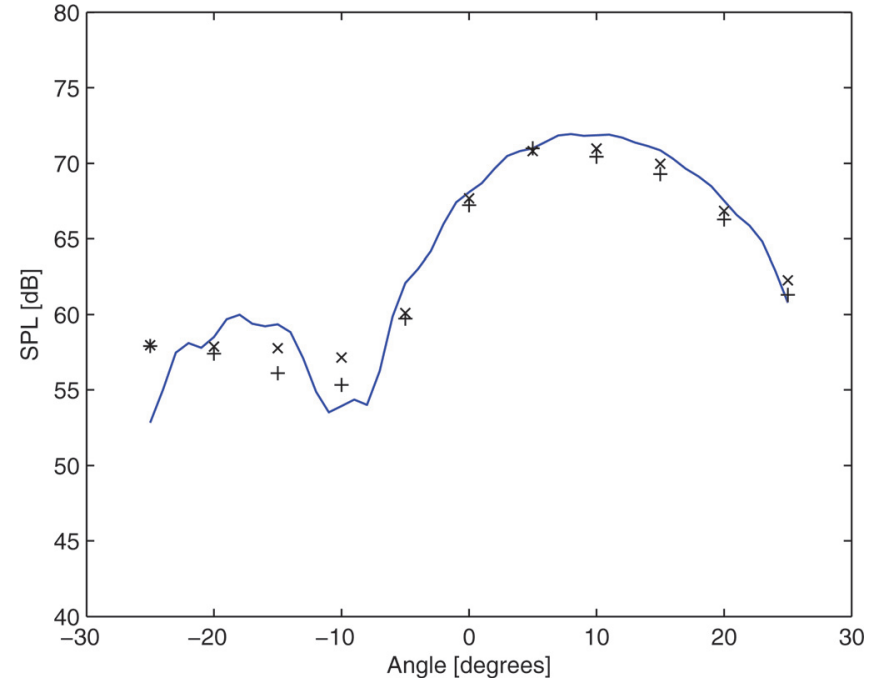

FIG. 5. (Color online) SPL in the transmitted field at $x=-0.46$. Loudspeaker positioned at $p=2, q=1 .+\times$ Experimental; $\longrightarrow$ theoretical.

have been of no practical significance, but in one or two tests, the occasional reading near the extremities of the measurement arc did drop below $50 \mathrm{~dB}$ such that these values could have been compromised by the influence of background noise. Measurements of sound pressure level on the transmitted side were also undertaken with the orifice section of the baffle plate filled in, in order to measure sound transmission through the supposed rigid baffle plate. The recorded levels varied with both loudspeaker and microphone position but generally were $10-15 \mathrm{~dB}$ below the levels recorded for similar positions with an open aperture. However, the difference did fall as low as $5 \mathrm{~dB}$ at the extremities of the measurement arc, even when the sound pressure levels recorded here were $10 \mathrm{~dB}$ or more above the background noise level.

In summary, the accuracy of the measured results given below is possibly compromised wherever the recorded level falls beneath $50 \mathrm{~dB}$ and generally toward the extremities of the arc of measurement.

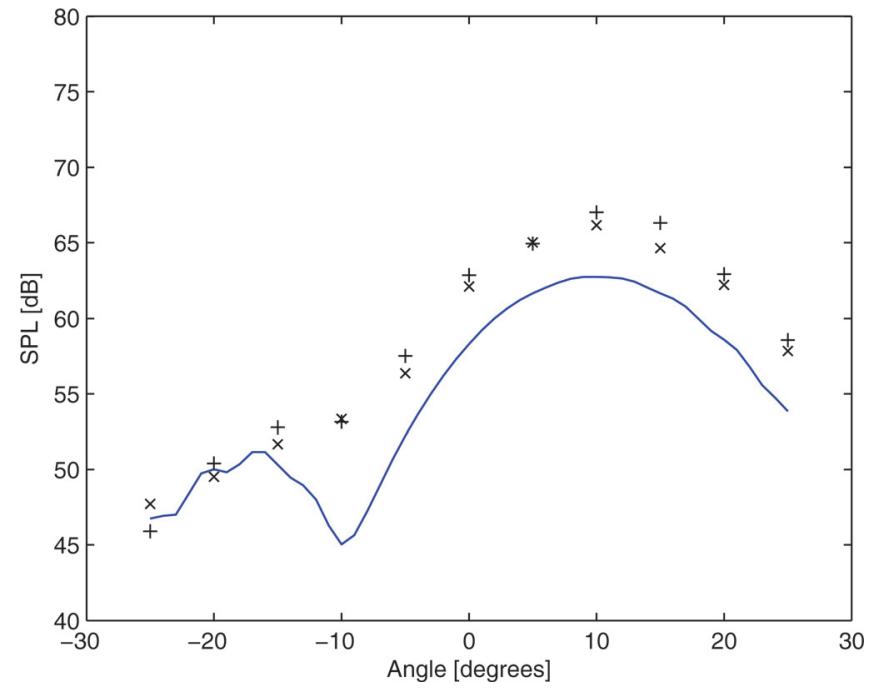

FIG. 6. (Color online) SPL in the transmitted field at $x=-1.29$. Loudspeaker positioned at $p=2, q=1 .+\times$ Experimental; $\longrightarrow$ theoretical. 


\section{RESULTS}

The first set of results, Figs. 3-6, correspond to integer $p, q$ values, or source locations chosen such that the source is tuned to drive mode $p, q$ within the aperture at cut-on. Figures 3-5 refer to a vertical plane of the measurement microphone which is at $x=-0.46 \mathrm{~m}$, whereas Fig. 6 refers to $x=-1.29 \mathrm{~m}$. Note that in all cases, the source and microphones are vertically beneath the center of the orifice. Figures 3 and 4 correspond to modes that are symmetric in $y$, namely $(1,0)$ and $(2,0)$, respectively, for which the source was placed in plane $y=0$. It is seen that the experimental results, particularly in the former case, are not precisely symmetric which is, therefore, indicative of experimental error. Furthermore, the close agreement between the two sets of experimental results indicates that there is some systematic experimental error due to asymmetry in the setup. This error becomes particularly noticeable near the angular limit set by the presence of the external doors, and the most probable cause of this would be differences in the absorbent lining applied to the face of these doors. Figure 5 gives similar results for an asymmetric mode, namely $(2,1)$. Discrepancies between experimental and theoretical results remain at a similar level as for the symmetric modes and could therefore be attributed solely to experimental error. The discrepancies are seen to increase as the measured sound level reduces, which happened to coincide with large angles for the two previous cases, and thus maybe in part due to reduced signal-to-noise ratio.

Similar trends to those evident in Fig. 5 are also to be seen in Fig. 6, where the vertical plane of measurement is further from the orifice center. In general, the discrepancies between theoretical and experimental results are larger, but then the measured values are all now lower such that there is an increase in error due to the reduced signal-to-noise ratio. However, in all of these figures for integer $p, q$ values the trend of the pressure distribution is clearly the same in both the theoretical and experimental results, while the precise sound levels are similar if not perfectly in agreement. The discrepancies observed could justifiably be due solely to

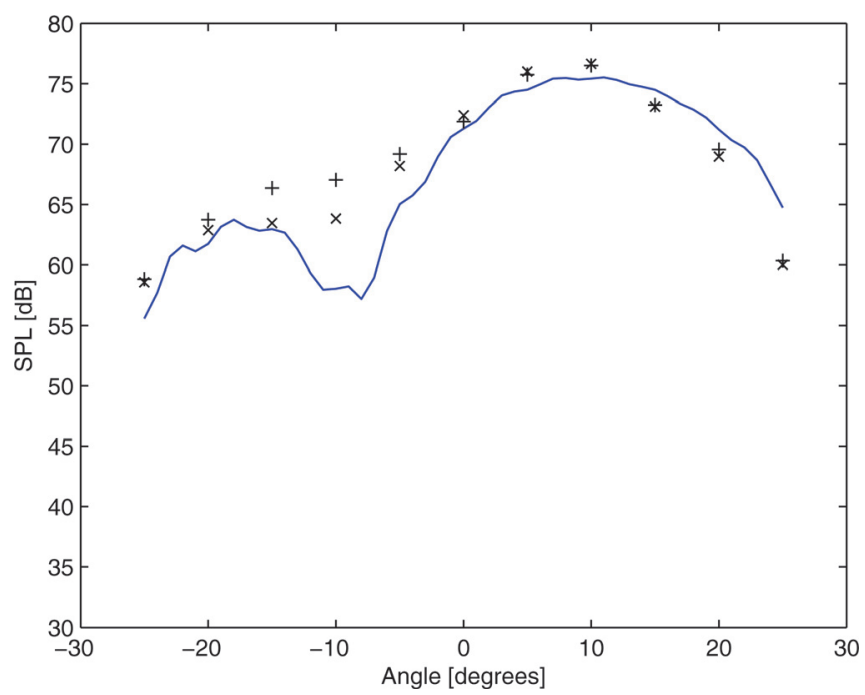

FIG. 7. (Color online) SPL in the transmitted field at $x=-0.46$. Loudspeaker positioned at $p=1.54, q=1.03 .+\times$ Experimental; $\longrightarrow$ theoretical.

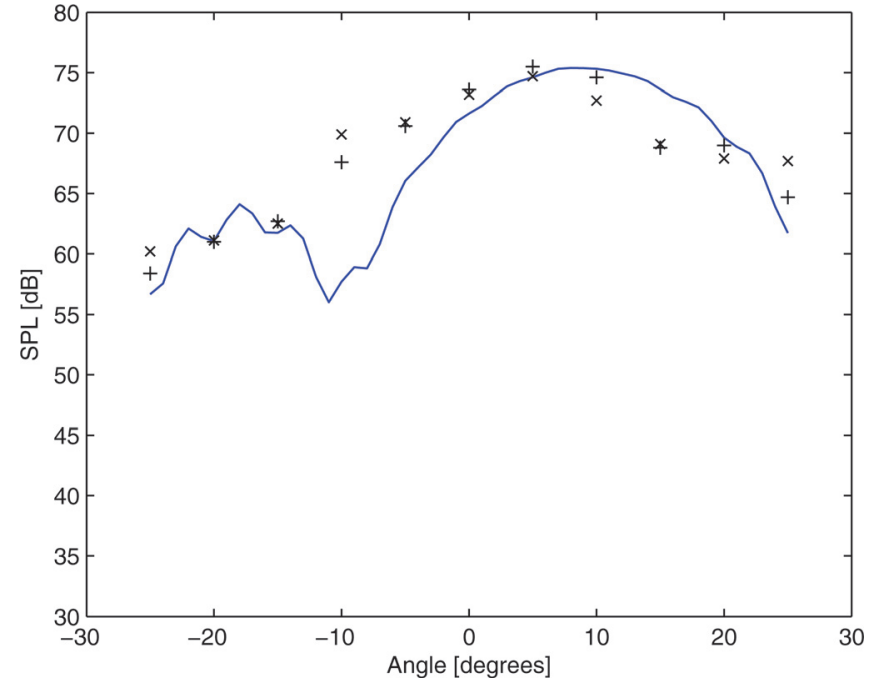

FIG. 8. (Color online) SPL in the transmitted field at $x=-0.46$. Loudspeaker positioned at $p=0.96, q=0.96 .+\times$ Experimental; $—$ theoretical.

experimental error and there is no specific cause to doubt the accuracy of the results from the approximate theory. Previous analytical studies of the approximate theory ${ }^{6}$ lead one to expect that it should be accurate under these conditions.

The results shown in Figs. 7-9 are of even greater practical significance in that they refer to non-integer $p, q$ values, where the source is located at random rather in a position to drive one particular mode strongly at cut-on. Figure 7 refers to $p=1.54, q=1.03$, with the measurement plane at $x=-0.46$. Similar results for the same $p$ and $q$ values were obtained for the measurement plane $x=-0.94$. Thus, the $q$ value is close to an integer but the $p$ value is almost midway between integer values. Similar results for $p=0.96$ and $q=0.96$ are shown in Fig. 8, such that now both $p$ and $q$ are close to but not exactly integer values. Finally, Fig. 9 refers to the case of $p=1.0, q=0.5$, where $p$ is exactly an integer and $q$ lies precisely midway between integer values. In all cases, it is observed that the discrepancies between theoretical and experimental values are no greater than for the cases

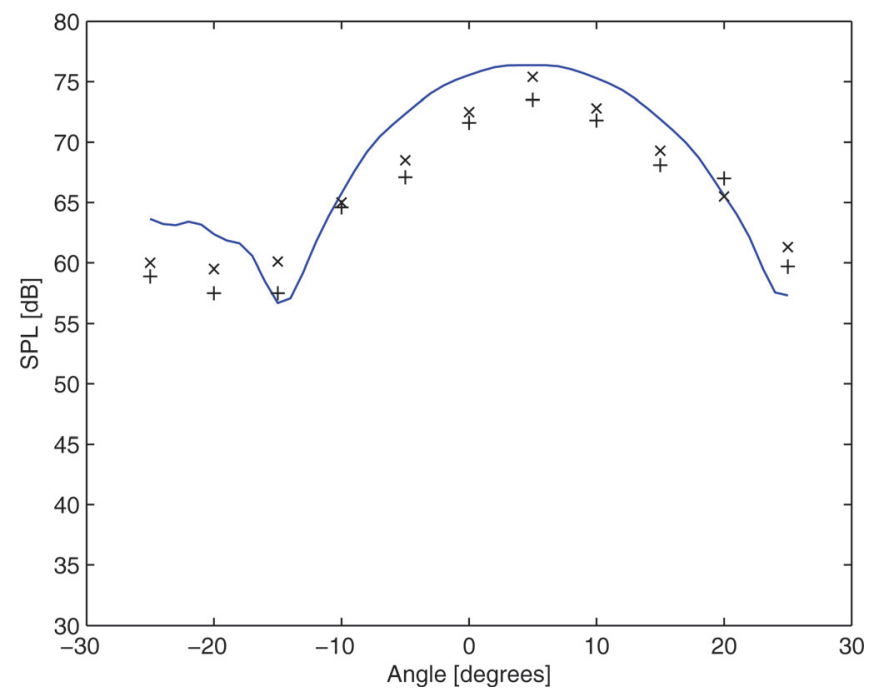

FIG. 9. (Color online) SPL in the transmitted field at $x=-0.94$. Loudspeaker positioned at $p=1.0, q=0.5 .+\times$ Experimental; $\longrightarrow$ theoretical. 
of integer $p, q$ values. Again, the trends are in very good agreement and the sound pressure levels are similar, with any differences within feasible bounds of experimental error. Earlier analytical work ${ }^{6}$ on the error analysis of the approximate theory did not extend to non-integer $p, q$ values, and thus, these findings are of particular significance, as they indicate that the approximate theory remains accurate for any general source location, provided that a number of higher-order modes are present in the aperture.

\section{CONCLUSIONS}

An approximate uncoupled mode solution to sound transmission through a rectangular aperture of finite thickness has been extended from the case of plane-wave impingement to that of impinging waves from a point source. The uncoupled analysis allows a solution technique that requires minimal computational effort as compared to a fully coupled analysis. It had been shown previously by analytical investigations that the uncoupled analysis gave accurate solutions provided that the impinging plane-wave sound field excited a mode in the aperture precisely at cut-on. The development to consider impinging sound from a point source has enabled experimental validation of the uncoupled analysis and it has been shown to yield accurate results for prediction of the transmitted sound field whether or not an aperture mode is driven at cut-on, or in other words for any relative positioning of the source and aperture. The technique does require a number of higher-order modes to be cut-on in the aperture.

${ }^{1}$ A. Sauter, Jr. and W. W. Soroka, "Sound transmission through rectangular slots of finite depth between reverberent rooms," J. Acoust. Soc. Am. 47(1), 5-11 (1970).

${ }^{2}$ P. M. Morse and K. U. Ingard, Theoretical Acoustics (Princeton University Press, Princeton, NJ, 1986), Chap. 9.

${ }^{3}$ K. Honga and H. Serizawa, "Diffraction of an acoustic plane wave by a rectangular hole in a infinitely large rigid screen," J. Acoust. Soc. Am. 106, 29-35 (1999).

${ }^{4}$ A. D. Pierce, R. O. Cleveland, and M. Zampoli, "Radiation impedance matrices for rectangular interfaces within rigid baffles: Calculation methodology and applications," J. Acoust. Soc. Am. 111(2), 672-684 (2002).

${ }^{5}$ H. H. Park and H. J. Eom, "Acoustic scattering from a rectangular aperture in a thick hard screen," J. Acoust. Soc. Am. (Lett.) 101(1), 595-598 (1997).

${ }^{6}$ J. L. Horner and K. S. Peat, "Approximations for the scattered field potential from higher mode transmission in rectangular apertures," J. Acoust. Soc. Am. 119(6), 3568-3576 (2006). 